\title{
Implementing a Lightweight Cloud-based Process Monitoring Solution for Smart Agriculture
}

\author{
Daniel Clarke, Hussain Al-Aqrabi $\left(\right.$ (凶) $^{[0000-0003-1920-7418]}$, Richard \\ Hill[ ${ }^{[0000-0003-0105-7730]}$, Pritesh Mistry, and Phil Lane ${ }^{[0000-0002-2179-0166]}$ \\ School of Computing and Engineering, University of Huddersfield, UK \\ \{h.al-aqrabi,r.hill,p.mistry,p.lane, \}@hud.ac.uk
}

\begin{abstract}
In order to meet recent challenges for more efficient and economic industrial manufacturing plants and processes, existing infrastructure is undergoing a digital transformation towards Smart Factories/Industry 4.0. These technologies and approaches also have applications outside of manufacturing, including agriculture. We introduce a fully integrated data analytics infrastructure that can be used to transfer and store relevant agricultural sensor data from microcontrollers. This is applied to a prototype plant monitoring system using a Raspberry Pi for data processing and an IoT Cloud system for Real Time Application. The prototype implementation of the microcontroller integrates a temperature sensor, a humidity sensor, and a capacitive moisture sensor. The design uses a standalone ESP32 micro controller communicating to an MQTT Broker using the publish/subscribe method. Sensor data can be accessed by subscribing to the MQTT topic or by using the Web Application. The ESPlantMonitoring web application is developed for user management to grant access to the MQTT broker and view collected sensor data.
\end{abstract}

Keywords: Internet of Things · Cloud Computing - Distributed Systems · Raspberry Pi. Arduino - Smart Agriculture

\section{Introduction}

The Internet of Things (IoT) refers to uniquely identifiable smart devices/objects connected to the internet that can sense data and react with their environment [1], [2]. Computing relies on the large amount of data being collected and made available by connecting all objects within an IoT system [4]. The IoT also relies on increasing developments in RFID (radio frequency identification), sensors, communication technologies, and IP [3], [5]. The fundamental concept is to have localised intelligent sensors to deliver the upper layers of applications without any ambiguity in human involvement [17].

The horticultural sector has previously depended on stand-alone embedded devices, including weather thermometers and sprinkler systems. Using sensor 
information and other external data, systems that were once stand-alone can be incorporated into larger scale systems and monitored to a higher precision [17]. The internet of things and cloud computing are driving introduction of Artificial Intelligence (AI) and robotics into farming [6]. 'Smart' farming not only targets agriculture on a large commercial scale [24], but can also be used to improve areas such as consumer-level agriculture. There are multiple examples of consumer-focused products such as GreenIQ and the Parrot Smart Pot[7]. Horticulture is a perfect candidate for an IoT smart device solution. The main factors affecting plant growth are easily observable by sensors, which makes monitoring easier to implement accurately.

The internet of things is being used within agriculture to help provide solutions for efficient farming [25]. An expanding worldwide population means that food production volume, and the efficiency of production, both need to increase. Climate change, soil quality and shrinking farmland availability all affect agriculture [22], [26]. A Microsoft project called FarmBeat is attempting to find a solution using artificial intelligence and the internet of things for data driven farming. Data is collected from sensors and drones before processing by machine learning algorithms to map the land. This data is used to facilitate precision agriculture, providing information to increase density, quality, and sustainability of the crops grown [8].

Limiting water use within agriculture is also important as it saves money. A real life implementation is SCADAfarm by WaterForce which offers multiple IoT farming solutions. Large scale farms use pivot irrigation systems to water crops. This is installed alongside already active irrigation systems. The sensor data is stored and processed in the cloud [10], [19], allowing irrigation control via a web or mobile application.

The sensors permit observation of the soil moisture level and set the watering depth. This is highly customised for different crops and farming layouts making it easier to avoid over-watering and optimising plant growth [9].

Several researchers have worked on automatic water sprinkling or irrigation. To determine the soil condition and water quantity, they opted for different metrics [13][15][14][16].

We examined various sensors and microcontrollers. This sophisticated system was a low-power device, with remote control and wireless network sensor technology, that can reduce manual conversion to remote automation [11]. We propose an automatic irrigation system to improve water use for plants in agriculture. A soil moisture sensor has been developed to assess soil water content. The system was tested in a sage crop field. The web application is designed to visualise results [12].

\subsection{Motivation}

In today's digital world, many farmers still use traditional methods. Digital technology allows farmers and agribusinesses to develop resilience, scale-up and sustainable solutions. Major efforts are being made to improve daily life via the introduction of smart devices and the internet of things. Using smart devices can 
help in many areas from trivial devices such as smart kettles to security systems. In the area of household horticulture, environmental factors greatly affect the growth of plants. Monitoring these factors can aid plant health and can also be used for home automation. Besides, The implementation of new technology in the field will solve major agricultural challenges.

\subsection{Contribution}

Our contribution to propose a lightweight cloud monitoring for smart agriculture. The system proposed comprises hardware as well as software. It also provides a platform to transfer and store the sensor data from microcontrollers. The microcontroller integrates a sensor of temperature and humidity as well as a capacitive sensor of moisture. Data is also stored in a SQL database, enabling information to be processed further. The Django framework is developed for user management to grant access to the MQTT broker and view collected sensor data.

Paper organisation - The remaining part of the paper is organisedas follows. In section 2, we discuss the proposed model. Section 3 describes the Raspberry Pi MQTT Broker and SQL Backup Implementation. Section 4, the ESPlantMonitoring web application is developed for user management to grant access to the MQTT broker and view collected sensor data in Section 5. Section $\mathrm{V}$ provides results and subsequent analysis, followed by MOTT Broker testing in Section 6. Finally, we conclude with directions for future development in Section 7 .

\section{Description of the model}

The proposed model comprises both hardware and software. The main system, implemented using a Raspberry Pi and microcontroller [20], is shown in the Fig. 1. The proposed IoT cloud for plant monitoring system using a Raspberry Pi comprises of an Internet of Things device (ESP32 microcontroller), Web Application (Django), Database (MYSQL), Web Server and a MQTT broker(Mosquitto) [21]. The Internet of Things device will collect data from multiple sensors. The device will be connected to the Internet via $\mathrm{Wi}-\mathrm{Fi}$ and will send the sensor data to the MQTT Broker. The database using MQTT publish. An MQTT broker is used to collect information from IoT sensors using MQTT publish, and easily accesses the live data using MQTT Subscribe. The MQTT broker will be running on a Raspberry PI [20], [23]. When the data is received via the Raspberry PI, it will be stored into the database by hooking into the publish event, converting to JSON and storing using MYSQL. The web application can be used to see previous sensor data stored and live data, while the Mobile Application is used to view live data from the MQTT Broker. Using the web application the user can register an account, this grants them access to the dashboard, within this dashboard users can add sensors using the unique key stored on the microcontroller. On registering, a new user will be added to the users table, this username/password is also used to access the MQTT server on the mobile application. 


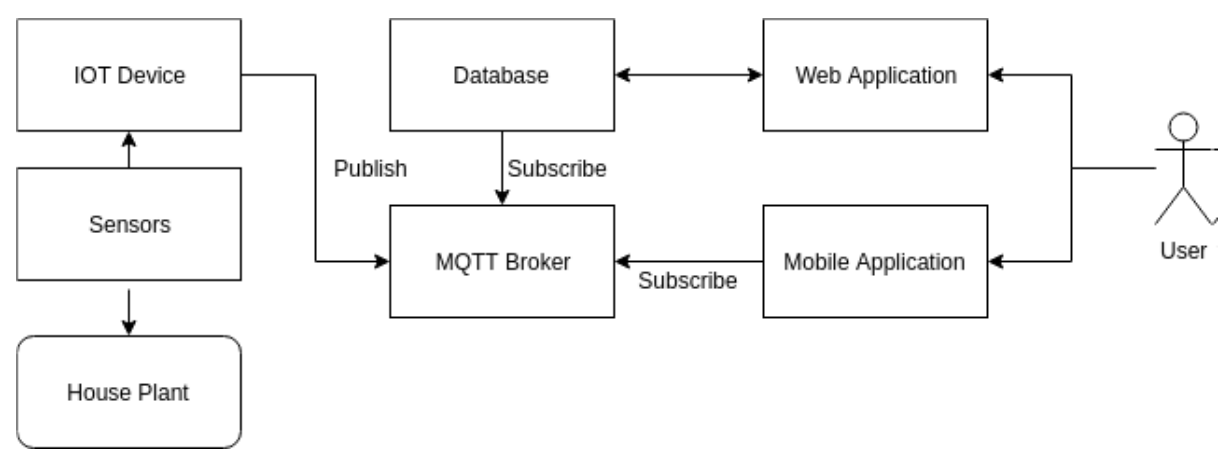

Fig. 1. Proposed Model.

\section{Raspberry Pi MQTT Broker And SQL Backup Implementation}

An MQTT broker is used to manage communication of MQTT messages using the publish-subscribe-based messaging protocol [21]. We chose Eclipse Mosquitto as the MQTT broker. This is a popular choice when configuring a MQTT broker on a Raspberry PI.

Alongside the main function of managing messages, we needed user authentication. This was done using the open-source plugin mosquitto-auth-plug.

\section{ESPlantMonitoring Homepage About $\quad$ Login Register}

\section{Welcome to ESPlantMonitoring}

This website provides a platform to collect soil moisture, temperature and humidity data of plants using ESP microcontrollers via the MQTT protocol.

Find out how to use the platform below.

\section{Learn more}

Fig. 2. ESPantMonintering Platform.

To set this up we need to compile both the ESPantMonintering Platform (Fig. 2), Mosquitto and the authentication plug-in code from source, using compati- 
ble versions. The Mosquitto auth plug-in is compatible with multiple different database back ends; we used MySQL.

- Password Hashing The plug-in provides authentication by checking the username and password against the relevant SQL table using a SQL query. The plugin requires the passwords to be stored using PBKDF2 hashing. This was important when developing the Web Application and affected the web development framework chosen to develop the application. Initially we were going to create the application using Laravel, but this would require a long process of modifying the middleware as the default hashing for Laravel is Bcrypt.

- Access Control List

Another security of the plug-in is the access control list this is a table within the database that defines which users can access which topic using wildcards. Without this feature users would be able to publish and subscribe to any topic they wanted, which would not be a secure way to manage the MQTT data.

- Mosquitto Configuration

The mosquitto config file is used to specify what settings the broker should use when running.

- SQL Backup

A key feature of the platform is the ability to view historical sensor data, Mosquitto does not provide database backup functionality, therefore we chose to write a Python script to store the sensor data. The script uses the Paho MQTT library to connect to the MQTT broker and subscribes to the relevant topic. It then retrieves the messages and deserializes the JSON and places it into an SQL statement. Finally the SQL statement is executed and the data is stored.

\section{ESPlantMonitoring Development}

The ESPlantMonitoring web application is developed for user management to grant access to the MQTT broker and view collected sensor data. Django is a web framework which follows the Model- View-Template architecture and is based on the Python programming language. Having the a large variety of libraries available, Django is a good choice for Web Applications. Interaction with the website changes depending on whether a user is logged in or not. The homepage/landing page gives a brief overview of what the platform provides and an option to learn more about the platform. Fig3 The About page gives a more in-depth description of the platform and gives details on how to setup ESP devices to interact with the platform. Fig4 In order to interact with the website, the user needs to register an account; this is done using the user login/register page. A valid account will have as a username an e-mail with valid syntax, and a unique password which is not similar to other details, and at least 8 characters which cannot be only numeric. 
Once a user is logged in, they can access the View Devices and user profile pages through the navigation bar. This page is the dashboard for managing devices, the main feature being to generate new keys. These keys are what links each unique device via the device id. All the users devices are then listed. Selecting the device will take you to the device details/management page. The device details page visualizes the data into a time-series chart for the user and gives the option to delete the device. Doing so will delete all relevant sensor data and remove the device id from their account.

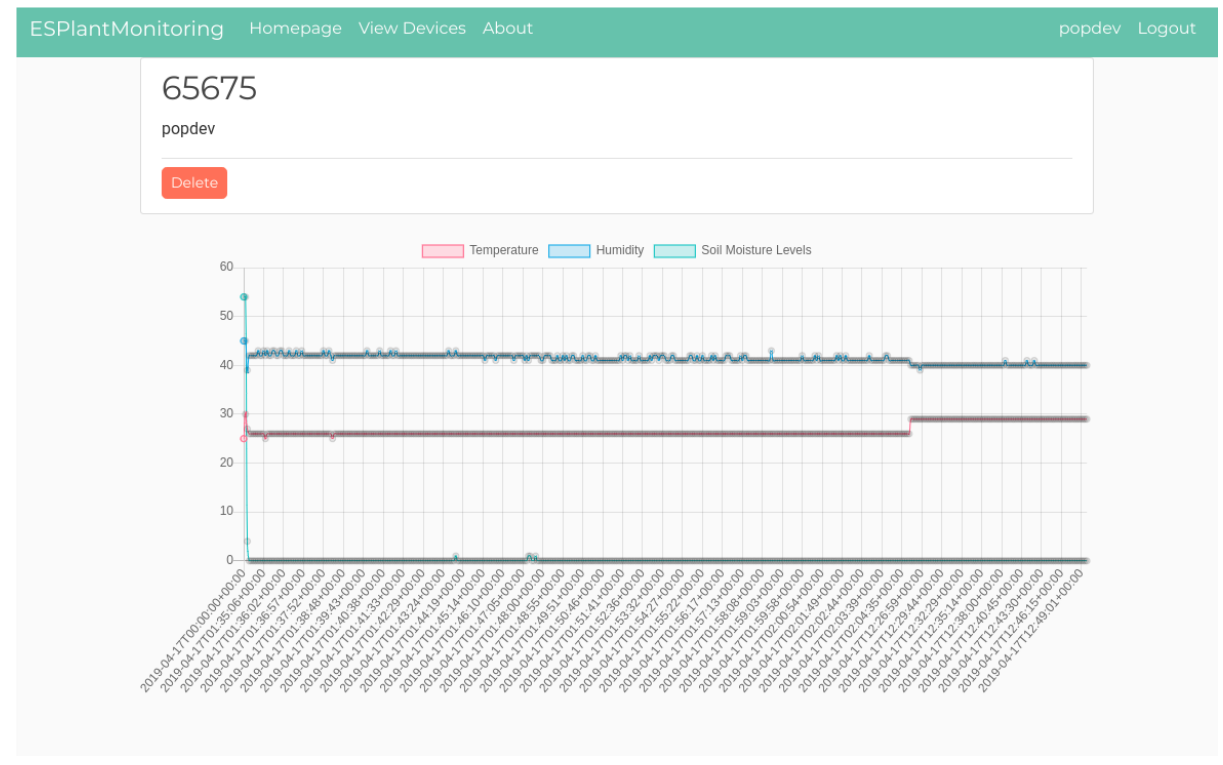

Fig. 3. Creating a new User.

The models define how the data in the database is structured and are used to interact with the data. Django has a built in user model, this creates the auth user table used to store user information. When a user is created they are added to the access control list, and allowed access to the topic sensors/username/. This is a topic that only they can access and use and should be used by all sensors. We then created two models called data and device. The device table stores user devices, linking the device id with the foreign key of user id from the users table. The data table then uses the device id as a foreign key to link devices with data taken from that sensor.

Django also creates multiple tables to structure and provide general admin functionality to the website. An important feature to remember when creating a web application that implements an SQL database is to setup links and restrictions, This prevents users from deleting data that will break the system. SQL creates links and restricions automatically on foreign keys. It is important to use 

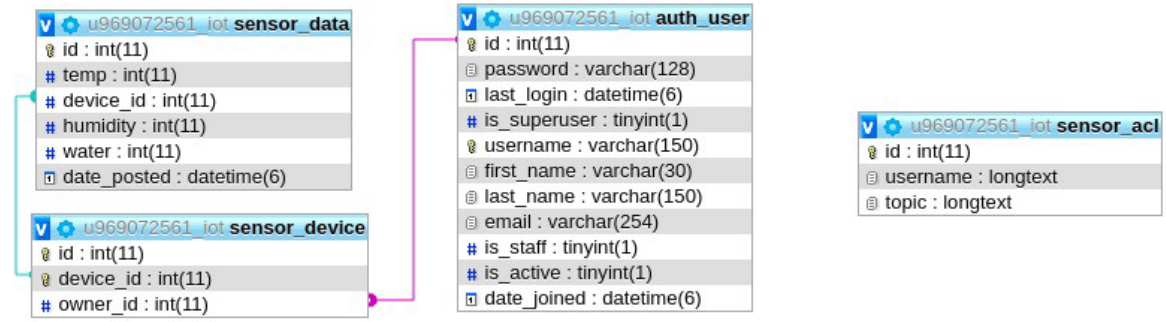

Fig. 4. User Authentication.

these links to determine what action is taken if a primary key used as a foreign key in another table is deleted. For example if a user deletes a sensor device, what happens to the linked sensors data. The SQL CASCADE method is used to delete data from child tables meaning that if the user a sensor then all linked data will be deleted also.

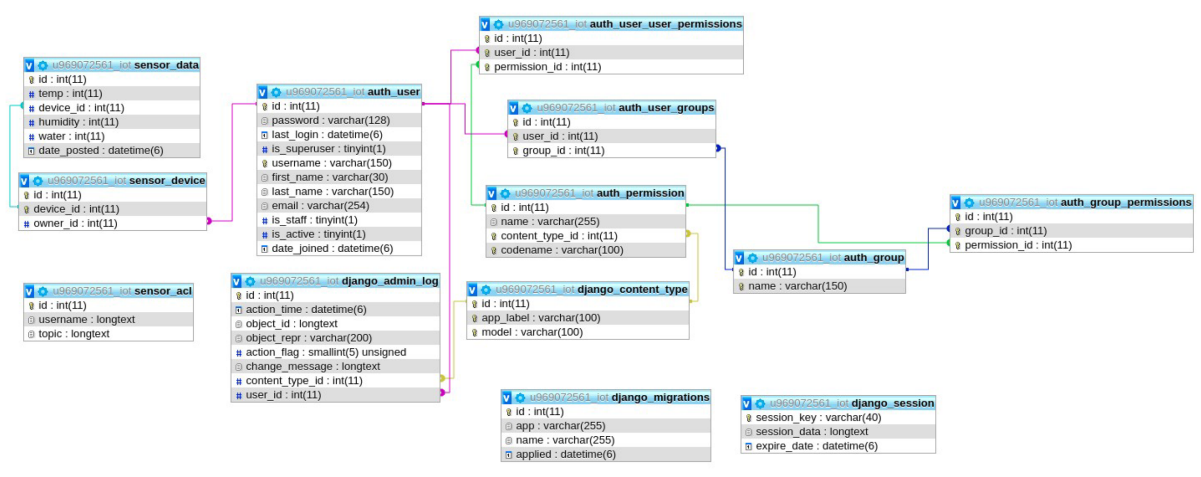

Fig. 5. Full Database Diagram.

A view function takes a web request and returns a HTTP response. This used for the management of site data and what is presented to the templates on certain pages. Within this work this HTTP response is usually a template alongside context variables. Covering each function within the view would be a large task which is not particularly relevant to the final product, therefore we restrict coverage to the functionality of managing and viewing devices and device data.

The sensor view implements the generic views provided by the Django framework, the views are linked to a selected model. ListView is used to list items within a certain model, Detail View is used to view information of the specified 
model item. Finally DeleteView is used to delete and confirm deletion of a model item from the database.

The generate function is used for the creation of devices. It generates a random number between 1 and 100000 . The value is then checked to be unique and stored in the database, returning the user to the page that the HTTP request was sent from. ListView is used to list items within a certain model, and DetailView is used to view information related to the specified model item. Finally DeleteView is used to delete and confirm deletion of a model item from the database.

The sensor view implements the generic views provided by the Django framework. These views are linked to a selected model. DateTime Handler is used to convert date time variables from SQL to JSON format. DeviceListView is used on the sensor homepage. Firstly the function retrieves the current logged in user, this makes it so the model items retrieved are user relevant. The DeviceDetailView requires context data for charting using the template. A query set is created taking the current user and Device ID selected. It the relevant data from the query set. This data is then converted to JSON format using the json.dumps function and placed into the context to be returned to the template. The DeviceDeleteView is used for the deletion of the selected device, the function check whether the device is owned by the user before confirming the deletion.

Templates represent how the user sees and interacts with the web application, using front-end web technologies including HTML, Bootstrap, and CSS. HTML is used to structure the website and Bootstrap and CSS are for visual representation. A free to use bootstrap template, called Minty from Bootswatch, was used mainly in the navigation bar and for the buttons. The views determine how to render information from the controller. We used the Chart.js JavaScript library to render visual charts using information collected from the controller. Data relevant to the selected sensor is displayed in time-series order with temperature, humidity and soil moisture as the datasets.

\section{Results and Analysis}

The prototype of the systems has been developed, and is deployed in a garden. The same prototype can also be used for agricultural applications conditioned by which sensor is required. Each sensor with its unique identity can access changes in the environment continuously. The test scenario of the prototype is shown in the figure. We used the serial monitor output to test sensor readings and check for errors. Once the device is switched on it attempts to connect to the WiFi Network specified, once connected to the network it will attempt to connect to the MQTT server and finally it will attempt to connect to the network time server.

The output shown in the red box is the configuration output, as can be seen all network connections were successful. The device places all relevant sensor information alongside the device ID and DateTime into JSON format, to test if the sensors are functioning correctly the data was printed to the serial monitor, 


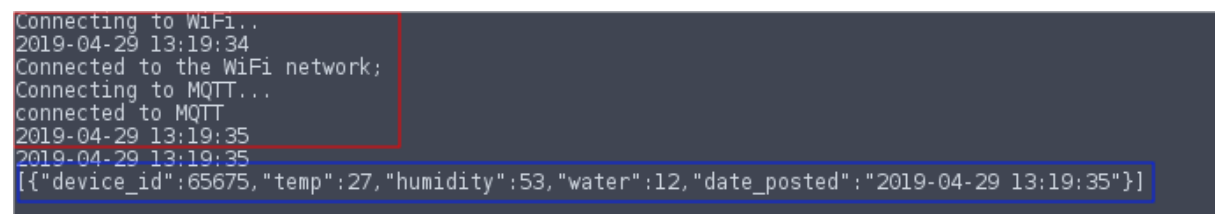

Fig. 6. IoT device is attempting to connect the MQTT server.

and this is shown in the blue box on the diagram. Each sensor produced a reading and was placed into the correct JSON format. To test sensor accuracy, we took a reading from the soil moisture sensor in a dry state and then fully submerged it in water to get two readings

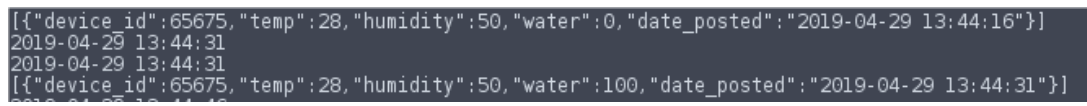

Fig. 7. Soil moisture sensor.

The calibration of the soil moisture sensor was correct, with a reading of 0 when dry and 100 when fully submerged. Unfortunately we were unable to verify the accuracy of the temperature and humidity sensor although this has no bearing on the demonstration of the usefulness of the system architecture.

\section{MQTT Broker Testing}

In order to test basic functionality of the MQTT broker we used a mobile application called MQTTool. This application provides connection testing and basic publish/subscribe functionality. Firstly, we tested if a user which is not specified in the auth user SQL table could connect to the public IP address. We then attempted to connect with an account within the table to the public IP address. The results were as expected and the broker authentication plug-in was working in this aspect.

Having determined the configuration of the ESP32 was correct and authentication worked, We then started the SQL backup script running on the Raspberry PI. we started the script and then powered the ESP32. The script prints the SQL statement derived from the sensor data. Firstly we compared the data from the serial monitor of the ESP32 to the SQL statement and then checked the statement was successfully executed to the database Comparing Figure 8 to Figure 9 shows that the JSON data was transferred and the correct SQL statement was produced. Figure 10 shows that SQL cursor executed the SQL statement correctly. The device details page is used manage devices, displaying device details and giving the option to delete the device. In order to test this functionality we populated the empty datasets shown in figure 8 via the IoT device. Once 
populated the chart is generated from the updated dataset shown in figure below. This data is shown in that database in figure below. The testing carried out shows the database working for sensor data from the IoT device and for device and user management. Improvements could be made by either changing database type from SQL or by monitoring the database over a period of time to find improvements to the efficiency. The MQTT broker was implemented to a certain extent,

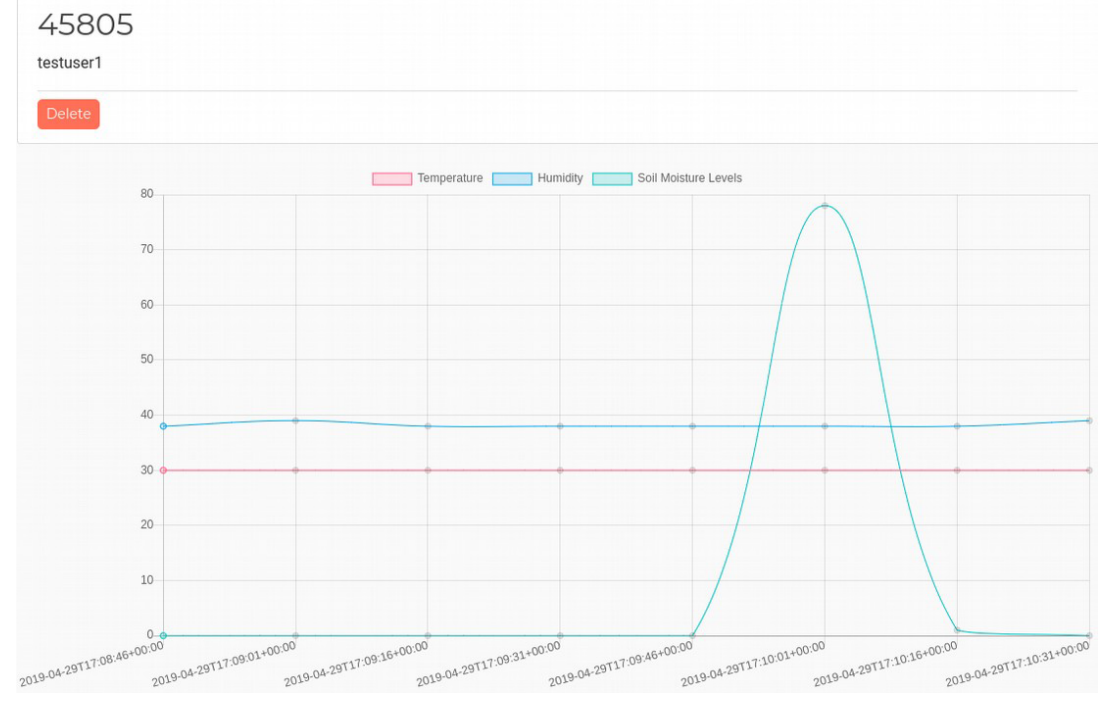

Fig. 8. User1 45805 sensor

In order to test this functionality, we populated the empty datasets via the IoT device. Once populated, the chart is generated from the updated dataset shown in figure 8 9. This data is shown in that database in figure 10 16. The testing carried out shows the database working for sensor data from the IoT device and for device and user management. Improvements could be made by either changing database type from SQL or by monitoring the database over a period of time to find improvements to the efficiency. The MQTT broker was implemented to a certain extent, general functionality worked and the SQL authentication was implemented.

The Web Application was fully functional, enabling user account and device managements once logged in. A user can have multiple sensors by using multiple unique device IDs through the device manager. Once devices are setup data is retrieved from the database and visualised in charts, this meets the requirements but with room for improvement, the charts show all data for the specified device, rather than displaying all data a better implementation would be to specify what time frame the data should be within. 


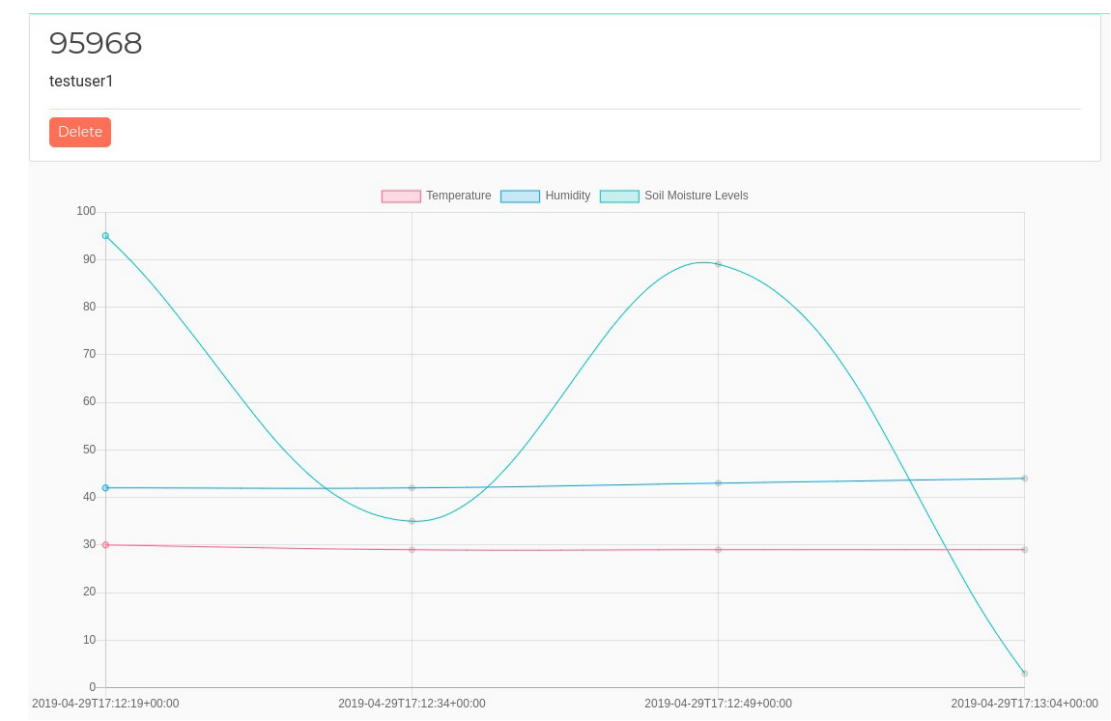

Fig. 9. User1 95969 sensor

\begin{tabular}{|r|r|r|r|r|r|}
\hline id & temp & device_id & humidity & water & date_posted \\
\hline 485 & 30 & 95968 & 42 & 95 & $2019-04-29$ 17:12:19.000000 \\
\hline 486 & 29 & 95968 & 42 & 35 & $2019-04-2917: 12: 34.000000$ \\
\hline 487 & 29 & 95968 & 43 & 89 & $2019-04-2917: 12: 49.000000$ \\
\hline 488 & 29 & 95968 & 44 & 3 & $2019-04-2917: 13: 04.000000$ \\
\hline
\end{tabular}

Fig. 10. 45805 SQL Data.

\begin{tabular}{|r|r|r|r|r|r|}
\hline id & temp & device_id & humidity & water & date_posted \\
\hline 485 & 30 & 95968 & 42 & 95 & $2019-04-2917: 12: 19.000000$ \\
\hline 486 & 29 & 95968 & 42 & 35 & $2019-04-2917: 12: 34.000000$ \\
\hline 487 & 29 & 95968 & 43 & 89 & $2019-04-2917: 12: 49.000000$ \\
\hline 488 & 29 & 95968 & 44 & 3 & $2019-04-2917: 13: 04.000000$ \\
\hline
\end{tabular}

Fig. 11. 95969 SQL Data. 


\section{Conclusions}

With the overwhelming generation of technology it is predicted that IoT has the ability to summon various technologies to allow new applications by agglomerating physical objects together in support of authentic, timely decision-making. This paper presents a prototype for a plant monitoring system using a Raspberry pi as a data processor and thus develops an IoT cloud system for real time applications.

An ESP8266 microcontroller enabled the development of the versatile IoT platform using integrated low-cost systems. The prototype implementation of the microcontroller integrates a temperature sensor and humidity sensor, and a capacitive moisture sensor. The ESPlantMonitoring web application is developed for user management to grant access to the MQTT broker and view collected sensor data.

\section{References}

1. A. J. Jara, M. A. Zamora-Izquierdo, and A. F. Skarmeta, Interconnection Framework for mHealth and Remote Monitoring Based on the Internet of Things, IEEE Journal on Selected Areas in Communications, vol. 31, no. 9, pp. 4765, 2013.

2. A. Ikram, et al. Approaching things (IoT): a Modelling, Analysis and Abstraction Framework, Concurrency and Computation: Practice and Experience, pp. 19661984, 2015.

3. H. Al-Aqrabi and R. Hill, Dynamic Multiparty Authentication of Data Analytics Services Within Cloud Environments, in 2018 IEEE 20th International Conference on High Performance Computing and Communications; IEEE 16th International Conference on Smart City; IEEE 4th International Conference on Data Science and Systems (HPCC/SmartCity/DSS), June 2018, pp. 742749.

4. M. Chui, M. Loffler, and R. Roberts, The Internet of Things, 2010.

5. K. Hwang and M. Chen, Big-data Analytics for Cloud, IoT and Cognitive Computing. John Wiley \& Sons, 2017.

6. Wolfert, S., Ge, L., Verdouw, C., \& Bogaardt, M.-J. (2017). Big Data in Smart Farming A review. Agricultural Systems, 153, 6980.

7. Parrot POT. (2017, November 29). Retrieved 27 Aug 2019, from Parrot Official website:https://www.parrot.com/global/connected-garden/parrot-pot

8. FarmBeats: AI, Edge \& IoT for Agriculture. (n.d.). Retrieved 27 April 2019, from Microsoft Research website: https://www.microsoft.com/enus/research/project/farmbeats-iotagriculture/

9. SCADAfarm - SOLUTIONS. (n.d.). Retrieved 27 June 2019, from https://www.scadafarm.com/solutions

10. H. Al-Aqrabi, L. Liu, R. Hill, N. Antonopoulos, A Multi-layer Hierarchical InterCloud Connectivity Model for Sequential Packet Inspection of Tenant Sessions Accessing BI as a Service. Proceedings of 6th International Symposium on (CSS) and IEEE 11th International Conference on (ESS). France, Paris, March 20-22, IEEE, 2014 pp. 137-144.

11. T. H.Gore, et al., Crop monitoring. Analysis and controlling system, Journal of Advanced research in computer science and software engineering, vol. 6, issue 2, February 2016. 
12. J. Gutierez, J. et al.,Automated Iriigation System using a Wireless sensor Network and GPRS Module, IEEE Transactions on Instrumentation and measurement,Vol.63,No.1, January 2014.

13. N.B. Bhawarkar, et al.Literature Review for Automated Water Supply with Monitoring the Performance System, International Journal of Current Engineering and Technology, Vol. 4, No. 5, Oct 2014.

14. S. S. Avatade, S. P. Dhanure, Irrigation System Using a Wireless Sensor Network and GPRS, International Journal of Advanced Research in Computer and Communication Engineering, Vol. 4, Issue 5, May 2015.

15. A. Koushik, et al. "Automatic drip irrigation system using fuzzy logic and mobile technology." Technological Innovation in ICT for Agriculture and Rural Development (TIAR), 2015 IEEE. IEEE, 2015.

16. B. Lala, et al. "Automatic crop irrigation system." Reliability, Infocom Technologies and Optimization (ICRITO), 2015 4th International Conference on. IEEE, 2015

17. A. M. Ezhilazhahi, \& P. T. V, Bhuvaneswari. (2017). IoT enabled plant soil moisture monitoring using wireless sensor networks. 2017 Third International Conference on Sensing, Signal Processing and Security (ICSSS), 345349.

18. S. Wolfert, L. Ge, C.Verdouw, M.-J. Bogaardt (2017). Big Data in Smart Farming A review. Agricultural Systems, 153, 6980.

19. H. Al-Aqrabi, L. Liu, R. Hill, L. Cui, J. Li, Faceted Search in Business Intelligence on the Cloud, in 2013 IEEE International Conference on Green Computing and Communications and IEEE Internet of Things and IEEE Cyber, Physical and Social Computing. IEEE, 2013.

20. ESP8266 vs. ESP32 on Battery Power. (2018, December 10). Retrieved 28 June 2019, from ESP8266 vs. ESP32 on Battery Power website: https://blog.voneicken.com/2018/lp-wifiesp-comparison/

21. HTTP vs. MQTT: A tale of two IoT protocols (2018). Retrieved 28 April 2019, from Google Cloud Blog website: https://cloud.google.com/blog/products/iotdevices/http-vsmqtt-a-tale-of-two-iot-protocols /

22. R. Lea, M. Blackstock (2014). Smart Cities: an IoT-centric Approach. ACM International Conference Proceeding Series.

23. M. G Williams, A Risk Assessment on Raspberry PI using NIST Standards, December 2012.

24. I Mohanraj, Ashokumarb Kirthika, J Naren, Field Monitoring and Automation using IOT in Agriculture Domain, vol. IJCSNS, no. 6, June 2015

25. J. Gutirrez, et al, "Automated Irrigation System Using a Wireless Sensor Network and GPRS Module", IEEE Transactions On Instrumentation And Measurement, vol. $17,2017$.

26. C. Liu, W. Ren, B. Zhang, C. Lv, "The application of soil temperature measurement by $\operatorname{lm} 35$ temperature sensors", International Conference on Electronic and Mechanical Engineering and Information Technology, vol. 88, no. 1, pp. 1825-1828, 2011. 